\title{
Access Network Design for Optical Flow Switching
}

\author{
Guy Weichenberg $^{\dagger \star}$, Student Member, IEEE, Vincent W. S. Chan ${ }^{\dagger}$, Fellow, IEEE, \\ and Muriel Médard ${ }^{\ddagger}$, Senior Member, IEEE \\ Laboratory for Information and Decision Systems \\ Massachusetts Institute of Technology
}

\begin{abstract}
In this work, we consider access network design for the Optical Flow Switching (OFS) transport architecture [1]-[3]. Our work addresses the all-optical physical layer of the data plane in the context of tree-based networks. We consider the advantages and disadvantages of passive and active components-including optical amplifiers-and ultimately integrate these building blocks in the most economically attractive fashion for the required number of users.
\end{abstract}

\section{INTRODUCTION}

A recent, pragmatic shift within the optical networking research community has led to increased emphasis on the economic viability of future network design. In particular, objectives of such research have related to the design of networks with excellent scalability: a decreasing cost per user, per unit of traffic, as the number of users and individual user bandwidth demand increase.

Advances and breakthroughs in optical devices and systems notwithstanding, electronics has remained the clear choice with which to carry out logical operations at network nodes. Thus, economically viable network architectures for the foreseeable future will necessarily incorporate electronic and optical technologies. Network designers are therefore faced with the task of judiciously (i.e., economically) integrating optical and electronic technology into capable network architectures.

In our previous work [4], [5], we conducted a throughputcost comparison of several prominent network architectures that incorporate varying degrees of electronics and optics: Optical Flow Switching (OFS), Tell-and-Go (TaG), Electronic Packet Switching (EPS), and Generalized Multi-Protocol Label Switching (GMPLS). These works indicated that, given presentday cost structures, OFS is the most scalable architecture in that it is most cost-efficient when the average user data rate is high and the aggregate network bandwidth demand is large. In reaching our conclusions, it should be noted that sensiblethough not necessarily optimal-access network designs were assumed for the architectures considered. We define an access network as the union of the metropolitan-area network (MAN) and local-area network (LAN) environments.

In this work, we address the data plane physical layer design of a cost-efficient access network suited to OFS. Since OFS is an all-optical end-to-end service, our access network design will use all-optical networking components exclusively ${ }^{1}$ for the data plane. In spite of this restriction, there is a wide gamut of optical network components at our disposal for this task.

\footnotetext{
${ }^{1}$ In long-haul transport, regeneration of optical signals is often required. At the moment (early 2007), electronic regeneration is a more practical alternative to all-optical regeneration. Nevertheless, we omit a consideration of electronic (and optical) regeneration from our present discussion, as we assume that any signal regeneration occurs in the wide-area network (WAN).
}

Our objective is to employ a subset of these components in such a manner that the requirements of OFS are met, while attempting to minimize the cost of the network.

\section{OFS OVERVIEW}

Owing to space constraints, we present only a high-level overview of OFS and refer the interested reader to [1]-[3] for further details. In OFS, users request end-to-end lightpaths for long duration (i.e., greater than $100 \mathrm{~ms}$ ) transactions. In order to schedule data transmission across the WAN, users communicate via an electronic control plane with the scheduling processors assigned to their respective MANs. These scheduling processors, in turn, coordinate transmission of data across the WAN in an electronic control plane. In OFS, it is assumed that the smallest granularity of bandwidth that can be reserved across the core is a wavelength. In the event that several single users have transactions which are not sufficiently large to warrant their own wavelength channels, they may multiplex their data for transmission across the WAN via dynamic broadcast group formation.

Motivated by the minimization of network management and switch complexity in the network core, flows are serviced as indivisible entities. That is, data cells comprising a flow traverse the network contiguously in time, along the same wavelength channel (assuming no wavelength conversion), and along the same spatial network path. This is in contrast to packet switched networks, where transactions are broken up into constituent cells, and these cells are switched and routed through the network independently. Note that in OFS networks, unlike packet switched networks, all queuing of data occurs at the end users, thereby obviating the need for buffering in the network core. A core node is thus equipped with a bufferless optical cross-connect (OXC). OFS is a centralized transport architecture in that coordination is required for logical topology reconfiguration. However, OFS traffic in the core will likely be efficiently aggregated and sufficiently intense to warrant a quasi-static logical topology that changes on coarse time scales. Hence, the centralized management and control required for OFS is not expected to be onerous. The network management and control carried out on finer time-scales will be distributed in nature in that only the relevant ingress and egress access networks will need to communicate.

We close this section by emphasizing that OFS is an architecture that is envisioned to serve high-end users with very large bandwidth demands exclusively. Users with comparatively small bandwidth demands will more efficiently be served by architectures such as EPS or GMPLS. Thus, the access networks proposed in this work are envisioned as playing a 
complementary role to other access architectures in the broader context of a hybrid network.

\section{NONAMPLIFIED OFS NETWORK DESIGN}

\section{A. Modeling assumptions}

In this section, we investigate the properties of access networks based upon tree topologies that do not employ optical amplifiers. The tree networks that we study are regular of degree $\Delta$; that is, each node, with the exception of the leaf nodes, has one parent node and $\Delta-1$ child nodes. Note that in our model, the root node also has a parent node- the WAN. We index each successive level, or stage, of the tree $i=0,1,2, \ldots$ beginning with the root node. An example tree network, with level indices indicated, is shown in Figure 1(a). We restrict our attention to tree topologies because they are known to be capable of supporting many users [6]. Furthermore, other common topologies (e.g., star, bus) are special cases of trees.

Figure 1 also depicts the two node architectures that we will study. To highlight the differences between the architectures, consider an inbound optical signal from the WAN on one of $w$ possible wavelength channels, which is destined for a particular node in the access tree network. In the passive node architecture shown in Figure 1(b), a small amount of optical power is tapped and subsequently processed locally at the node, and the remaining power is split evenly among the $\Delta-1$ child nodes by means of a passive star coupler (PSC). In order to enable any two nodes in the tree to communicate with one another, we will assume a PSC of dimensions $\Delta \times \Delta$. In the active node architecture drawn in Figure 1(c), all of the desired wavelength channel power is (ideally) directed, via an all-optical switch, directly to the local node or to one of the $\Delta-1$ child nodes. In order to enable any two nodes in the tree to communicate with one another, we assume that the active switch is realized using a $w(\Delta+1) \times w(\Delta+1)$ OXC; that is, the $w$ wavelength channels on a fiber are (de)multiplexed (before) after the active switching occurs at wavelength granularity. These two node architectures are by no means exhaustive. Indeed, an intermediate node architecture employing a subset of the above passive and active elements may also be possible. The arrayed waveguide grating (AWG) is another candidate component for an all-optical node. Like active switches, AWGs localize optical power to output ports and do not suffer from inherent splitting loss. AWGs, however, are wavelength-selective devices in that they route different wavelength channels of an input signal to different output ports. Furthermore, unlike active switches such as microelectro-mechanical systems (MEMS) which are reconfigurable, AWGS are passive devices that are statically configured, which permits them to be far less expensive than active switches. The combination of their wavelength-selective and static natures results in a static partitioning of wavelength resources among the different outputs. This is an unattractive property for highly dynamic traffic or for traffic that cannot be forecasted accurately at the time of node deployment. Owing to these significant shortcomings of AWGs, we omit their consideration as candidate building blocks for all-optical nodes.

In addition to the splitting loss inherent to some optical devices (e.g., PSCs), we account for fiber loss due to material

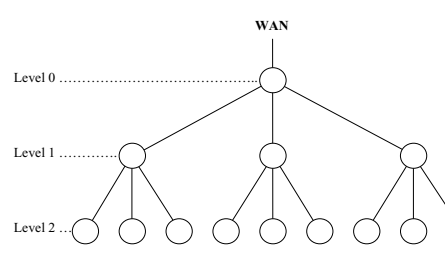

(a)

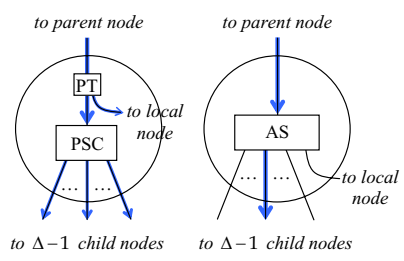

(b)

(c)
Fig. 1. (a) A tree in which $\Delta=3$. (b) A passive node employs a passive tap and a PSC. (c) An active node employs an active switch (AS).

absorption and Rayleigh scattering, and excess loss of components due to manufacturing and design nonidealities. Note that in the absence of excess loss, it is clear that the active node architecture will support the largest number of nodes, owing to the absence of inherent splitting loss. Indeed, it is only the fiber attenuation of the optical signal that will limit the number of users supported by this architecture under this idealized condition. We shall, however, account for the excess loss of optical components in our analysis since, i) excess loss can be significant, and ii) excess loss is typically more significant in active components than in passive components, thus diminishing the perceived advantage of node architectures employing active components in lieu of passive components which suffer from inherent splitting losses.

\section{B. Homogeneous network analysis}

We now analyze homogeneous tree architectures-tree networks which comprise exclusively of one of the aforementioned two node types. In our analyses, we will often decouple the wavelength channels in a fiber and treat them independently. We are able to do this because intrachannel performance impairments, such as stimulated Brillouin scattering (SBS) and self-phase modulation (SPM), often limit system performance before interchannel impairments, such as stimulated Raman scattering (SRS) and cross-phase modulation (CPM), take effect.

Defining $P_{i n}^{i}$ and $P_{\text {out }}^{i}$ to be the input and output wavelength channel powers, respectively, of a node at the $i$ th level of the tree, we have the following relationships for the passive and active node trees, respectively:

$$
\begin{gathered}
P_{\text {out }}^{i}=P_{\text {in }}^{i} \frac{\left(1-\alpha_{p}^{i}\right)\left(1-\delta_{p}\right)}{\Delta}=P_{\text {out }}^{i-1} \frac{\left(1-\alpha_{p}^{i}\right)\left(1-\delta_{p}\right)(1-l)}{\Delta} \\
P_{\text {out }}^{i}=P_{\text {in }}^{i}\left(1-\delta_{a}\right)=P_{\text {out }}^{i-1}\left(1-\delta_{a}\right)(1-l)
\end{gathered}
$$

where $\alpha^{i}$ is the fraction of power tapped for local processing at an $i$ th level node, $\delta$ is the excess loss from the parent input port to a child (i.e., nonlocal) node output, and $l$ is the fiber attenuation between successive levels which is assumed to be constant. In general, $\delta$ and $l$ are not 'free' parameters, but are dependent upon other network parameters, such as $\Delta$ and $i_{\max }$, respectively. As a result, we will employ the notation $\delta(\Delta)$ and $l\left(i_{\max }\right)$ to emphasize these dependencies.

For the passive node architecture, we generally allow for the possibility of different tap values $\alpha^{i}$ at each stage. Using a customized tap value at each level such that only the minimal amount of optical power required for detection, $P_{\text {sens }}$, 
is bled off maximizes the number of supportable usersalbeit at increased complexity. Regardless of the types of taps employed, the above insertion loss relationships lead to a recursive analysis which is summarized next.

1) Passive nodes: For the case of passive nodes, it can be shown that:

$$
P_{\text {out }}^{i}=\frac{1}{1-l\left(i_{\max }\right)}\left[P_{\text {in }}^{i-k} b^{k+1}-P_{\text {sens }} b\left(\frac{1-b^{k+1}}{1-b}\right)\right],
$$

for $k=0,1, \ldots, i$, where $b=\frac{\left[1-\delta_{p}(\Delta)\right]\left[1-l\left(i_{\max }\right)\right]}{\Delta}$ represents the insertion loss across one level of the tree. The optimum tap value $\alpha_{o p t}^{i}$ is given by:

$$
\alpha_{o p t}^{i}=\frac{P_{\text {sens }}}{P_{\text {in }}^{i}}=\left[b^{i} h-b \frac{1-b^{i}}{1-b}\right]^{-1},
$$

where $h=P_{i n}^{0} / P_{\text {sens }}$-known as the head-room-represents the maximum number of supportable broadcast users in the absence of excess and fiber loss. The maximum supportable tree level index $i_{\max }$ is solved to be:

$$
i_{\text {max }}=\left\lfloor\frac{-\log (h-h b+b)}{\log b}\right\rfloor,
$$

and the maximum number of supportable users is thus given by:

$$
N_{\max }=\sum_{i=0}^{i_{\max }}(\Delta-1)^{i}=\frac{1-(\Delta-1)^{i_{\max }+1}}{2-\Delta} .
$$

In Figure 2(a), we plot the maximum number of supportable users and the maximum number of tree levels as a function of node degree for the passive node architecture with optimum tap values. In the presence of an excess loss per stage that is independent of $\Delta$, one would expect the optimal tree topology to reduce to a star, which has indeed been noted in [6]. However, as we see in Figure 2(a), even in the presence of an excess loss per stage that scales logarithmically (in $\mathrm{dB}$ ) with $\Delta$, the optimal topology still reduces to a star.

2) Active nodes: For the active node case, the recursive relationship obtained above applies after substituting $\alpha=0$ and omitting the inherent splitting loss:

$$
P_{\text {out }}^{i}=P_{\text {in }}^{i-k}\left[1-l\left(i_{\max }\right)\right]^{k}\left(1-\delta_{a}[w(\Delta+1)]\right)^{k+1} .
$$

The maximum supportable tree level index $i_{\max }$ is solved to be:

$$
i_{\text {max }}=\left\lfloor\frac{-\log h-\log \left(1-\delta_{a}[w(\Delta+1)]\right)}{\log \left(1-\delta_{a}[w(\Delta+1)]\right)+\log \left[1-l\left(i_{\max }\right)\right]}\right\rfloor
$$

and, as before, the maximum number of supportable users is:

$$
N_{\max }=\frac{1-(\Delta-1)^{i_{\max }+1}}{2-\Delta} .
$$

In contrast to Figure 2(a) for the passive node architecture, Figure 2(b) for the active node architecture indicates that the star topology is not optimal. Indeed, under our assumptions, the maximum number of supportable users is attained for $\Delta \approx 450$, and is roughly 250,000 -well beyond the envisioned number of OFS subscribers in a given access network. The reason that the number of supportable users declines beyond $\Delta \approx 450$ is that, in this regime, the excess loss of an active switch becomes so large that a cascade of smaller switches can support the same number of users with less loss.
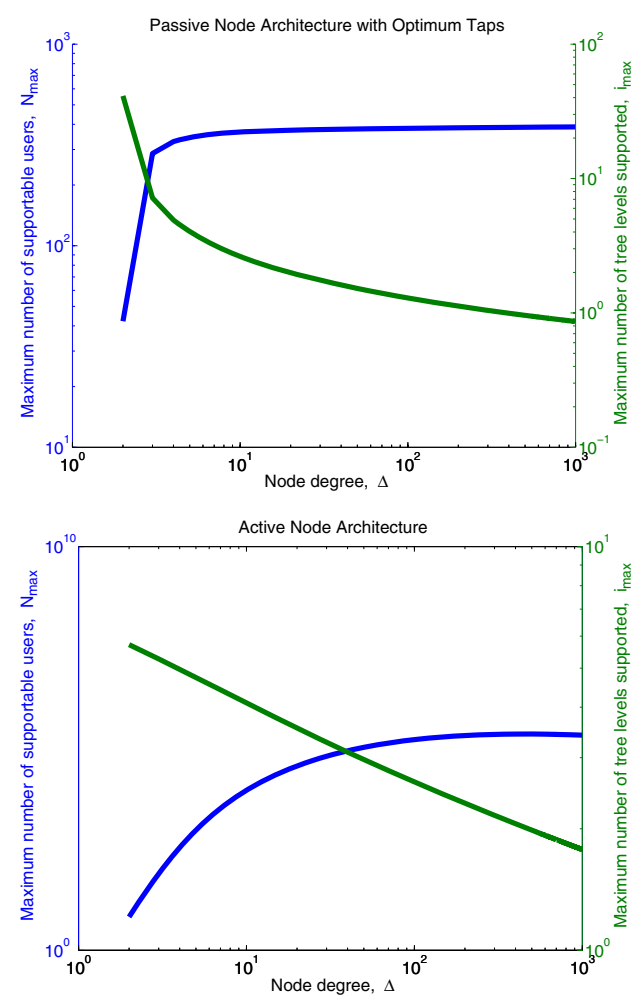

Fig. 2. Maximum number of supportable users $\left(N_{\max }\right)$ and maximum number of supportable tree levels $\left(i_{\max }\right)$ as a function of node degree $(\Delta)$ for the passive node architecture with optimum tap values (a), and the active node architecture (b). Realistic loss assumptions on optical components are made, including excess loss on optical switching/splitting devices that scales logarithmically (in $\mathrm{dB}$ ) with the number of ports. In addition, $h=1000$, $w=10$, and integrality constraints on $N_{\max }$ and $i_{\max }$ are neglected.

\section{Mixed architectures}

In the previous subsection, we observed that a nonamplified tree network comprising exclusively passive nodes cannot support more than a few hundred users, whereas a nonamplified tree network comprising active nodes can support many millions of nodes. A natural question then is how one should construct a network required to serve an intermediate number of users-say 50,000 users. One solution is to construct a tree network using active nodes exclusively. This approach has the drawbacks that i) it will likely lead to an expensive network design owing to the presence of active components in every internal tree node, and relatedly, ii) it may result in the tree network's leaf nodes wastefully receiving far more optical power than needed for detection. We are therefore led to wonder whether it is possible to trade some number of supportable users and/or abundant optical power for lower network cost. Indeed, less power-efficient passive nodeswhich are expected to be less expensive than hybrid or active nodes - can be substituted to reduce the network cost.

We present now the Mixed-A architecture, an example of which is drawn in Figure 3. Here, the upper levels of the tree comprise active nodes, and the lowermost level of the tree comprises passive star networks of $N_{p}$ end-users. In contrast to other possible mixed architecture designs, we do not allow for groups of local users to hang off internal active nodes. This restriction is motivated by the fact that in many real networks, nodes housing active switches are exclusively used as 'relays'. 


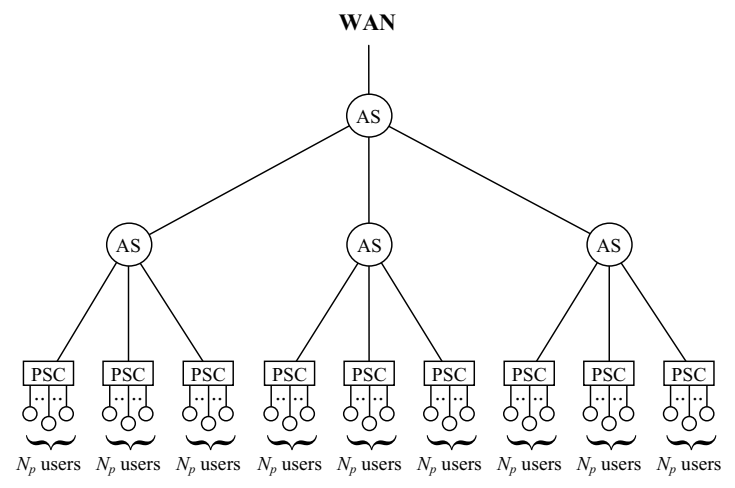

Fig. 3. An example of the Mixed-A tree architecture in which $\Delta=4$ and $i_{m i x}=2$. The number of end-users supported is $(\Delta-1)^{i_{m i x}} N_{p}=9 N_{p}$.

Consequently, in the Mixed-A architecture, the active switch residing at each active node has dimensionality $w \Delta \times w \Delta$.

1) Analysis: We simplify the foregoing analysis by assuming that the passive portion of the network will sustain all of the fiber loss. This is reasonable since our design process will be inclined towards minimizing the number-and hence geographic span-of active nodes. Then, for successful detection we require that the wavelength channel power entering each coupler, $P_{i n}^{p}$, respect the following inequality:

$$
P_{i n}^{p} \geq \frac{P_{\text {sens }}\left(N_{p}+1\right)}{(1-l)\left[1-\delta_{p}\left(N_{p}+1\right)\right]} .
$$

The maximum number of tree levels of active nodes, $i_{\text {mix }}$, can be shown to be:

$$
i_{m i x}=\left\lfloor\frac{-\log h^{*}}{\log \left[1-\delta_{a}(w \Delta)\right]}-1\right\rfloor
$$

where we employ a modified head-room definition:

$$
h^{*}=\frac{P_{i n}^{0}}{P_{i n}^{p}}=h \frac{P_{\text {sens }}}{P_{i n}^{p}},
$$

which ensures that each PSC is fed a sufficient amount of power for the $N_{p}$ end-users to successfully detect. The total number of end-users supported by the architecture is then:

$$
N_{\max }=(\Delta-1)^{i_{\operatorname{mix}}} N_{p} .
$$

\section{Cost-driven Mixed-A architecture}

In this subsection, we search for the design parameters $\Delta$, $i_{m i x}$, and $N_{p}$ that minimize the network cost per end-user, given that the network must support a predetermined minimum number of nodes $N_{r e q}$. Our cost model considers only initial capital expenditure of a network, and neglects ongoing operational costs, which may constitute a significant portion of a network's cost. Among the relevant capital expenditure costs, the fiber cost is particularly difficult to model as it can range from being exorbitant as in the case of green-field networks, to being almost insignificant as in the case of brown-field networks. Furthermore, a large part of the variability of this cost is attributable to commercial and political factors. Other capital expenditure costs, such as transceivers, are omitted in our model as they are independent of the design parameters $\Delta$, $i_{m i x}$, and $N_{p}$ and will thus manifest as a constant cost offset.
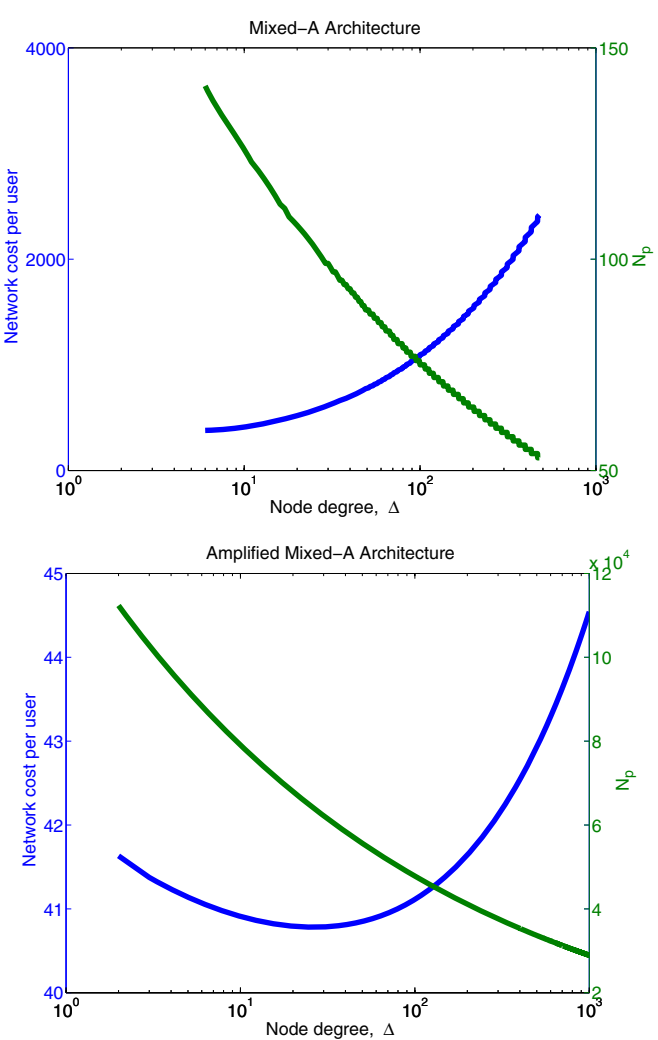

Fig. 4. Network cost per end-user node $\left(C_{\operatorname{mix}} / N_{\max }\right)$ and number of passive nodes per PSC $\left(N_{p}\right)$ as a function of active node degree $(\Delta)$ for the cost-optimal Mixed-A architecture with $N_{r e q}=50,000$. The integrality constraint on $i_{m i x}$ is neglected. The previous excess loss scaling assumptions are made, in addition to: $h=1000, G=1000, w=10, C_{a m p}=10^{5}$, $\beta_{3 D}=10^{3}, \beta_{3 d B}=10, \beta_{f}=0$, and $\epsilon=1.3$.

Thus, the salient cost components of the Mixed-A architecture that we explicitly account for are:

- Active switch cost. The cost of an OXC is well modeled by a convex function of the number of switch ports, where the nature of the convex function is governed by the architecture of the switch fabric [7]. In our study, we will assume that active switches are realized with a 3dimensional MEMS architecture with the following cost model: $C_{a s}(k)=\beta_{3 D} k^{\epsilon}$, where, as of today, $\beta_{3 D} \approx$ $\$ 1000$, and $1 \lesssim \epsilon \lesssim 1.5$.

- PSC cost. We assume that a $k$-port PSC is formed by cascading $\log _{2} k$ stages of $\frac{k}{2} 3 \mathrm{~dB}$ couplers. We will therefore model the cost of a $k$-port PSC as

$$
C_{p s c}(k)=\beta_{3 d B} \frac{k \log _{2} k}{2},
$$

where, as of today, $\beta_{3 d B} \approx \$ 10$.

Consequently, the aggregate network cost, as a function of $\Delta$, $i_{m i x}$, and $N_{p}$, is:

$C_{m i x}=\frac{1-(\Delta-1)^{i_{m i x}}}{2-\Delta} C_{a s}(\Delta)+(\Delta-1)^{i_{m i x}} C_{p s c}\left(N_{p}+1\right)$,

for the Mixed-A architecture.

In Figure 4(a), we plot for each value of $\Delta$ the normalized network cost and $N_{p}$ of the cost-optimal design when $N_{r e q}=$ 50,000 end-users need to be supported. We observe from Figure 4(a) that the minimum cost of the Mixed-A architecture 
(\$379.70/end-user) — 2 orders of magnitude less than the cost of the analogous network comprising active nodes only-is achieved when $\Delta=5$. We remark, however, that the loss and cost scaling functions play a critical role in determining the parameters that minimize the network cost. If, for example, we employed a linear cost scaling function for the active switches, then the optimal active portion of the network would be more inclined towards a star. We also note that there is an inverse relationship between the two quantities plotted on the ordinate axes, suggesting that a cost-efficient design minimizes (maximizes) the amount of active (passive) equipment.

\section{AMPLIFIED OFS NETWORK DESIGN}

In this section, we consider the use of optical amplifiersspecifically, the erbium-doped fiber amplifier (EDFA) - in the Mixed-A architecture as a means to further lower the cost of the network. The optimal placement of optical amplifiers in a general network is a complex problem that has been considered extensively in the literature. Intuitively, the placement of an optical amplifier in a network should permit a large gain to be extracted from the amplifier, while attempting to minimize the degradation of signal quality as a result of the inevitable introduction of amplified spontaneous emission (ASE) noise. In the specific context of the Mixed-A architecture, we choose to position optical amplifiers between the active and passive stages of the network, as shown in Figure 5. In the appendix, we justify this decision from the perspectives of both the 'down-link' and 'up-link' directions of data flow. Our rationale, however, does not preclude the use of other/additional optical amplifiers to further reduce the network cost.

The analysis of the amplified Mixed-A architecture is identical to that of its nonamplified counterpart, provided that the modified headroom parameter $h^{*}$ is replaced with the parameter $\hat{h}=G h^{*}$, which reflects the per-wavelength amplifier gain. Such an analysis is valid provided that equation (2) in the Appendix is respected. Note that this analysis assumes that the signal entering the root node of the amplified MixedA architecture is clean (i.e., does not contain ASE noise). In the case that this signal does contain previously injected ASE noise, an upper bound on the amplifier gain may exist.

In Figure 4(b), we plot for each value of $\Delta$ the normalized network cost and $N_{p}$ of the cost-optimal design when $N_{r e q}=50,000$ end-users need to be supported. The same cost structure as $C_{m i x}$ is employed, with the added term $(\Delta-$ $1)^{i_{m i x}} C_{a m p}\left(N_{p}+1\right)$, representing the cost of the amplifiers. The minimum cost of the amplified Mixed-A architecture (\$40.78/end-user) is achieved when $\Delta=26$. Note that this normalized cost is an order of magnitude less than the cost of the corresponding optimal nonamplified Mixed-A architecture. The significant cost savings arose from the amplifiers enabling an increase in the number of passive users of two orders of magnitude. In doing so, the number of expensive active switch ports in the network was reduced, at the smaller expense of increasing the size of the PSCs and installing amplifiers.

\section{CONCLUSION}

In this work, we considered the design of the data plane physical layer of an OFS access network. The physical topolo-

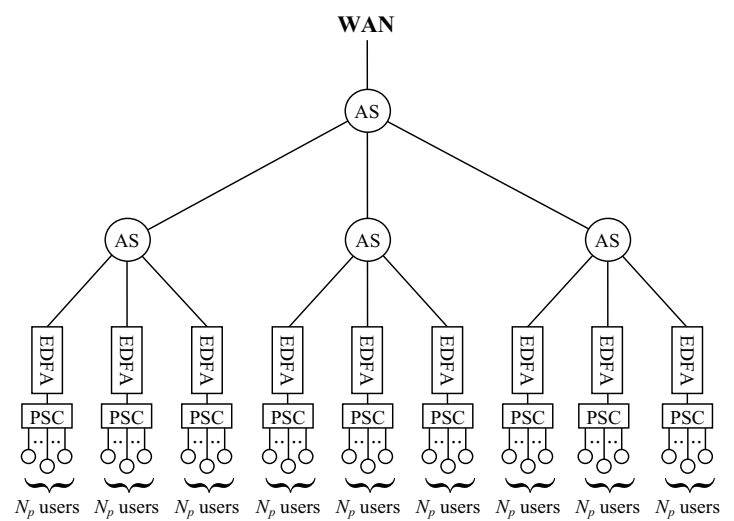

Fig. 5. An example of the Mixed-A tree architecture with amplification.

gies that we investigated were based on regular trees. Analyzing the tradeoffs between the use of passive and active components within tree topologies, we proposed a mixed architecture appropriate for serving an intermediate number of users, and demonstrated that optical amplification can significantly lower the cost of the access network.

\section{APPENDIX}

\section{A. Direct detection of optically amplified signals}

When a signal is passed through a single optical amplifier, the amplified signal power incident at the photodetector can be written as:

$$
P_{a m p}=G P_{s}+P_{s p}
$$

where $G$ is the amplifier gain, $P_{s}$ is the input optical signal power, and $P_{s p}=(G-1) n_{s p} \bar{h} v \Delta v_{s p}$ is the spontaneous emission noise power added to the signal, in which $n_{s p}$ is the spontaneous emission factor, $\bar{h}$ is Planck's constant, $v$ is the optical frequency, and $\Delta v_{s p}$ is the effective bandwidth of spontaneous emission which can be approximated by the amplifier bandwidth.

The variance of the photocurrent fluctuations at the detector can be written as:

$$
\sigma^{2}=\sigma_{T}^{2}+\sigma_{s}^{2}+\sigma_{s p-s p}^{2}+\sigma_{s i g-s p}^{2}+\sigma_{s-s p}^{2}
$$

corresponding to thermal noise, signal shot noise, spontaneous-spontaneous beat noise, signal-spontaneous beat noise, and spontaneous emission shot noise, respectively, where:

$$
\begin{aligned}
\sigma_{T}^{2} & =\frac{4 k_{B} T F_{n}^{R} \Delta f}{R_{L}} \\
\sigma_{s}^{2} & =2 q\left[\mathcal{R}\left(G P_{s}+P_{s p}\right)+I_{d}\right] \Delta f \\
\sigma_{s p-s p}^{2} & =4 \mathcal{R}^{2}\left[(G-1) n_{s p} \bar{h} v\right]^{2} \Delta v_{o p t} \Delta f \\
\sigma_{s i g-s p}^{2} & =4 \mathcal{R}^{2} G P_{s}(G-1) n_{s p} \bar{h} v \Delta f \\
\sigma_{s-s p}^{2} & =4 q \mathcal{R}(G-1) n_{s p} \bar{h} v \Delta v_{o p t} \Delta f
\end{aligned}
$$

where $k_{B}$ is Boltzmann's constant, $F_{n}^{R}$ is the noise figure of the pre- and main amplifers, $\Delta f$ is the effective electrical bandwidth, $T$ is the absolute temperature, $R_{L}$ is the load resistor, $q$ is the electronic charge, $\mathcal{R}$ is the responsivity of the detector, $I_{d}$ is the dark current, $n_{s p}$ is the spontaneous 
emission factor, and $\Delta v_{\text {opt }}$ is the optical bandwidth of the spontaneous-emission noise.

For an optically amplified signal which is shot noise limited at the amplifier's input and signal-spontaneous beating noise limited at the output, the noise figure is given by:

$$
F_{n} \equiv \frac{\mathrm{SNR}_{\text {in }}}{\mathrm{SNR}_{\text {out }}}=\frac{2 n_{\text {sp }}(G-1)}{G} .
$$

Neglecting $\mathcal{R} P_{s p}$ and $I_{d}$ in the previous shot noise equation and substituting in $F_{n}$ and $\mathcal{R}=\eta q / \bar{h} v$, where $\eta$ is the quantum efficiency of the detector, the previous noise power component equations can be written as:

$$
\begin{aligned}
\sigma_{T}^{2} & =\frac{4 k_{B} T F_{n}^{R} \Delta f}{R_{L}} \\
\sigma_{s}^{2} & =\frac{2 q^{2} \eta G P_{s} \Delta f}{\bar{h} \nu} \\
\sigma_{s p-s p}^{2} & =\left(q \eta G F_{n}\right)^{2} \Delta v_{o p t} \Delta f \\
\sigma_{s i g-s p}^{2} & =\frac{2(q \eta G)^{2} F_{n} P_{s} \Delta f}{\bar{h} \nu} \\
\sigma_{s-s p}^{2} & =2 q^{2} \eta G F_{n} \Delta v_{o p t} \Delta f .
\end{aligned}
$$

\section{B. Amplifier placement justification in Mixed-A architecture}

1) Down-link: In the down-link direction, placing the amplifiers just before the PSC allows for a large gain to be extracted from the amplifier partly because the signal is weak at this point, having passed through a series of active switches. The previous sections, furthermore, indicated that the key to a cost-efficient architecture is a large number of users connected to each PSC (i.e., large $N_{p}$ ). A cost-efficient architecture will consequently possess a large splitting loss - on the order of $20 \mathrm{~dB}$ or more-after passing through the PSC, implying that the signal power prior to the PSC needs to be well-above the thermal noise-limited receiver sensitivity. Provided that this is indeed the case, we now show that the impact of the added ASE noise is small.

Let us denote the wavelength channel signal power just prior to the amplifier by $P_{i n}^{a m p}$, and the channel signal power just after the amplifier by $G P_{a m p}^{i n}$, where $G$ is the amplifier gain of the wavelength channel assuming operation of the amplifier in the linear regime. For a thermal noise-limited analysis to be appropriate for detection of the signal at the end-user, we require that the received channel signal power at each end-user exceed the thermal noise-limited receiver sensitivity $P_{\text {sens }}^{t}$. In addition, we also require that the signal-spontaneous beat noise-which we assume to be the dominant source of noise outside thermal noise-be dominated by the thermal noise when the signal power is at the thermal noise-limited receiver sensitivity. These two conditions reduce to:

$$
P_{i n}^{a m p} \begin{cases}\geq & \frac{P_{\text {sens }}^{t}\left(N_{p}+1\right)}{G\left[1-\delta_{p}\left(N_{p}+1\right)\right](1-l)} \\ \gg & \frac{\left(q \eta P_{\text {sens }}^{t}\right)^{2} F_{n} R_{L}}{2 \bar{h} \nu k_{B} T F_{n}^{R}} .\end{cases}
$$

Assuming parameter values which are consistent with typical, present-day optical transmission systems, and defining:

$$
g \equiv 10 \log \left[\frac{G\left[1-\delta_{p}\left(N_{p}+1\right)\right](1-l)}{N_{p}+1}\right]
$$

(i.e., amplifier gain net, of excess, splitting, and fiber loss in dB) results in the following constraints:

$$
P_{i n}^{a m p}\left\{\begin{array}{l}
\geq-(30+g) \mathrm{dBm} \\
\gg-60 \mathrm{dBm} .
\end{array}\right.
$$

For typical values of amplifier gain $G$ and excess loss, and (desirable) values of $N_{p}$ of hundreds or greater, $P_{i n}^{a m p}$ would respect these constraints for the Mixed-A architecture. Hence, detection at end-users is indeed thermal noise-limited, implying that the added ASE noise will not impact performance appreciably in the down-link direction.

2) Up-link: In the up-link direction, a large PSC splitting loss, resulting from a large $N_{p}$, implies that a large gain can be extracted from the optical amplifier without the amplified signal subsequently incurring the deleterious effects of fiber nonlinearities. Furthermore, because the amplifier is placed as upstream as possible after the PSC, the impact of ASE noise is minimized in this direction. In order to ensure that the ASE noise is not significant compared to the thermal noise when the signal is detected downstream, the following condition, by similar reasoning as before, must be met:

$$
P_{u} \gg \frac{\left(q \eta P_{\text {sens }}^{t}\right)^{2} F_{n}\left(N_{p}+1\right) R_{L}}{2\left[1-\delta_{p}\left(N_{p}+1\right)\right](1-l) \bar{h} \nu k_{B} T F_{n}^{R}} .
$$

where $P_{u}$ is the up-link signal power launched by the enduser. Assuming typical, present-day values for the above parameters, this constraint becomes:

$$
P_{u} \gg\left(-60+10 \log N_{p}\right) \mathrm{dBm} \text {. }
$$

In order to meet a minimum desired signal power further upstream in the network, a launch power well in excess of a few milliwatts may be necessary in the Mixed-A architecture that we are considering. Such high launch signal powers are known to be problematic for long-haul transmission systems, as nonlinear effects have sufficient fiber distance to wreak havoc on the signal quality. The nonlinear effects most severely limiting the performance of optical systems are SBS and SPM. It can be shown that performance limitations arising from these nonlinear effects in the high data rate systems that we are considering can be virtually eliminated by constraining the distance from the end-user to the PSC to be on the order of tens or hundreds of meters.

\section{REFERENCES}

[1] V. W. S. Chan, K. L. Hall, E. Modiano, and K. A. Rauschenbach, "Architectures and technologies for high-speed optical data networks," IEEE/OSA Journal of Lightwave Technology, vol. 16, no. 12, pp. 21462168, Dec. 1998.

[2] B. Ganguly and V. W. S. Chan, "A scheduled approach to optical flow switching in the ONRAMP optical access network testbed," Proceedings of IEEE/OSA Optical Fiber Communication Conference, pp. 215-216, Mar. 2002.

[3] V. W. S. Chan, G. Weichenberg, and M. Médard, "Optical flow switching," Proceedings of the Workshop on Optical Burst Switching, Oct. 2006.

[4] G. Weichenberg, V. W. S. Chan, and M. Médard, "Cost-efficient optical network architectures," Proceedings of ECOC, Sept. 2006.

[5] — "On the throughput-cost tradeoff of multi-tiered optical network architectures," Proceedings of IEEE Globecom, Nov. 2006.

[6] V. W. S. Chan, S. Chan, and S. Mookherjea, "Optical distribution networks," Optical Networks Magazine, vol. 3, pp. 25-33, Jan. 2002.

[7] C. Guan and V. W. S. Chan, "Cost-efficient physical architecture for OXC-switched WDM mesh networks-(generalized) Moore graphs and their close relatives," Proceedings of IEEE Globecom, Nov. 2006. 